\title{
Artificial saliva in diabetes Type II: A Registry crossover study
}

\author{
Cornelli $\mathrm{U}^{1 *}$ and Belcaro $\mathrm{G}^{2}$ \\ ${ }^{1}$ Loyola University School of Medicine Chicago - 2160 First Ave Maywood IL \\ ${ }^{2}$ Irwin Labs University of Chieti- Strada Statale 16 Bis 94 Spoltore 65010 (PE) Italy
}

\begin{abstract}
Background: Xerostomia is a quite common symptom diabetes Type II.

Objective: To evaluate the activity of an artificial saliva spray (AS) in patients with diabetes Type II presenting xerostomia

Methods: Registry crossover study in 16 cases comparing AS with Tonimer throat (TT). Patients were following a three-day treatment with each product ( 3 times/ day). A period of three days washes out was followed between the two arms of the crossover. The symptoms under investigation were xerostomia (main variable measured with a ordinal scale from 0 to 6), stimulated salivary flow, and soluble antioxidant test (SAT) in saliva. At the end of the experience the patients were asked which treatment was considered the best.
\end{abstract}

Results: The AS was reducing xerostomia from $4.2 \pm 0.83$ to $1.7 \pm 0.6$ (ANOVA $\mathrm{p}<0.001$ ) and SAT from $3290 \pm 505.3$ to $2496 \pm 285.2 \mu \mathrm{mol} / \mathrm{L}$ whereas using TT the respective values were almost identical to the baseline values. The patrients expressed a clear preference with $97 \%$ in favor of AS.

Conclusion: In patients with diabetes type II AS is reducing the xerostomia and the inflammation of the oral tissue.

This trial was registered at clinicaltrials.gov as NCT03452085 ID IAPSSAPP

Abbreviations: $\mathrm{AS}=$ artificial saliva; $\mathrm{TT}=$ Tonimer throat spray; SAT $=$ saliva antioxidant test; UWS $=$ Unstimulated Whole Saliva; SWS = Stimulated Whole Saliva; BMI = Body Mass Index

\section{Introduction}

Xerostomia is a subjective complaint of dry mouth and is frequently associated to the reduction of the salivary flow $<0.1 \mathrm{~mL} / \mathrm{min}$ at rest (unstimulated salivary flow or UWS) or $<0.7 \mathrm{~mL} / \mathrm{min}$ under stimulation (stimulated salivary flow or SWS) [1].

The presence of xerostomia is frequent among patients suffering from Type I and Type II diabetes because the diseases are prone to dehydration and one of the effect is dryness of the mouth plus several other oral complications such as periodontal disease, candidiasis, and burning mouth sensation [2]. Diabetes causes polyuria, as a result of osmotic diuresis when the glucose levels are so high that glucose is excreted with urine. Polydipsia (excessive thirst), is a consequence of polyuria, and the latter can compromise the salivary flow.

In Type I and Type II diabetes a low salivary flow rate and higher xerostomia was shown compared to healthy control [3]. However, some author found that patients affected by Type II diabetes showed only a trend of salivary flow reduction, most probably in relation to the glycemic control [4]. Inadequate salivary gland function has been shown to be associated with abnormal glycemic control [5].

The maximal excretion rate of the four major salivary glands after stimulation was found reduced in diabetes Type II patients complaining xerostomia when compared with those without xerostomia [6]. Older adults with poorly controlled diabetes may have impaired salivary flow in comparison with subjects with better controlled diabetes [7]. However, several factors are capable of inducing salivary disorders in these patients such as aging, therapies and concomitant pathologies. A review of clinical studies done in the field, despite the large variation among studies, showed high prevalence of xerostomia in diabetes compared to normal population respectively in $12.5 \%-53.5$ $\%$ Vs $0-30 \%[1]$.

Recently it has been shown that an oral moisturizing jelly saliva substitute was effective in the relief of xerostomia in elderly patients with diabetes and hypertension, based upon subjective and objective dry mouth scores [8]. The treatment with moisturizing jelly needs a quite complex procedure (e.c. packaging to be opened, use of spoons, fingers to spread the product) and people do prefer a simpler system, as just a spray to be used throughout the day when needed.

The product that will be addressed in the present study consists of aldiamed $^{\circledast}$ spray which is an artificial saliva commonly available on the market as a Medical Device.

The product contains Aloe vera and was found very effective in vitro in case of tests considering the wetting and permanence on materials

Correspondence to: Umberto Cornelli, MD, PhD, Piazza Novelli 520129 MilanItaly, Tel: 3902714904; Email: ucornelli@gmail.com

Key words: xerostomia; diabetes type II; salivary flow; antioxidant capacity of saliva; aldiamed ${ }^{\oplus}$

Received: February 05, 2018; Accepted: February 13, 2018; Published: February 20, 2018 
similar to the oral tissue [9], and also particularly effective against the Candida albicans [10].

The present study was an open interventional Registry in adult subjects suffering from diabetes Tipe II and xerostomia. The experience was aimed to compare aldiamed ${ }^{\oplus}$ spray (AS) versus Tonimer throat spray (TT) in a 3-day crossover study. The limited period of time was chosen to minimize discomfort of xerostomia in case the treatments were not effective.

\section{Material and methods}

The study was conducted at a single center. It was designed and implemented according to UNI EN ISO 14155:2012 and to STROBE checklist [Strobe statement Version 4 as published in Oct/Nov 2007] in conformity with the guidelines of the Declaration of Helsinki.

\section{Experimental design}

The study follows a typical clinical pharmacology design in the form of a Registry study, conducted as a crossover scheme to compare two treatments AS and TT: all the subjects were treated with the two products under analysis.

\section{Subjects}

Twenty-one subjects that were part of the epidemiological screening for asymptomatic vascular diseases conducted in the Irwin Lab3 of Spoltore (PE-Italy).

\section{Admission Criteria}

Subjects aged 30 to 75 years, suffering from xerostomia and diabetes Type II since at least one year with stabilized oral hypoglycemic therapy from at least 6 months. The degree of xerostomia for the admission had to be at least $>2$ according to a semi quantal scale ranging from 0 to 6 (see variables). Concomitant chronic diseases pertaining to dyslipidemia, cardiovascular and/or gastrointestinal diseases, and anxiety/depression were accepted provided under adequate therapy since at least 6 months.

\section{Exclusion criteria}

Patients already under treatment for xerostomia or with xerostomia score $\leq 2$ were excluded. Subjects suffering from obesity (BMI $\geq 30$ $\mathrm{Kg} / \mathrm{m}^{2}$ ), cancer of any type, drug addiction and alcoholism were also excluded.

\section{Timetable}

The study was conducted according to 5 phases that started in September 2017 and were concluded in November 2017.

The first phase consisted in a preliminary selection of the individuals that was done during the screening phase of the epidemiological study (in the month of September). The selection consisted in asking the patients suffering from diabetes Type II about the presence of xerostomia (simply yes or no) and the willing to participate as volunteers to a study on this symptom.

The second phase was the first measure of xerostomia for all the cases that accepted to enter the study, followed by the random allocation of patients to one of the crossover arm.

The third phase was the development of the first arm of the crossover that lasted 3 days. The fourth phase was a wash out period of 3 days. The fifth phase was the development of the second arm of the crossover and lasted 3 days.

\section{Variables}

Four variables were considered: xerostomia was the main variable, whereas stimulated salivary flow, saliva antioxidant capacity (SAT), and the patient's preference for treatment AS or TT were respectively secondary variables.

\section{Xerostomia}

Xerostomia was considered the main variable and was measured according to a semi quantal visual scale (or VAS; visual analogue scale) ranging from 0 to 6 with intervals of 1 point as follows:

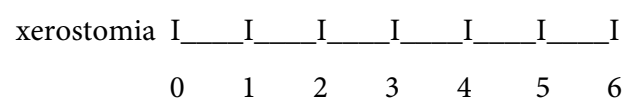

The subject had to indicate the degree of xerostomia and half a point of the scale was not permitted.

\section{Stimulated salivary flow}

The method used has been standardized previously [11]. In short, the subject was asked to chew a standard square of medical cotton of $150 \pm 20 \mathrm{mg}(2 \mathrm{~cm} \mathrm{x} 2 \mathrm{~cm})$ for $1 \mathrm{~min}$ rolling the cotton in the mouth in order to collect the saliva. In the minute about 60 chewing were requested $(1 / \mathrm{min})$ recommending to avoid the compression of the cotton with the tooth. The measure of the flow consisted in weighing the cotton before and after the chewing. The method became familiar to the subject after repeating the test a couple of times before starting the baseline flow evaluation.

\section{Antioxidant capacity of saliva (SAT test)}

The method that was used is standardized [12]. In short, the test utilizes the capacity of thiocyanate $(\mathrm{SCN})$ to react with iron $\left(\mathrm{Fe}^{3+}\right)$ causing the formation of $\mathrm{FE}\left[(\mathrm{SCN})_{6}\right]^{3-}$. This last compound is characterized by a brown-red color. The reducing elements contained in the fluid to be tested (saliva in this case) transform the $\mathrm{Fe}^{3+}$ into $\mathrm{Fe}^{2+}$ avoiding the reaction with the SCN and the consequent entity of the color. The variation was measured with a photometer at $505 \mathrm{~nm}$, and the normal value in saliva should be between $947-1459 \mu$ molar/L (taking vitamin $\mathrm{C}$ as reference value). The value exceeding the normal value can be interpreted in two ways: an abundant use of antioxidants or an inflammatory process in the oral cavity that generate vasodilation and consequent extravasation of plasma antioxidants.

The measure was taken always before breakfast (between 8 to 9 am). SAT was measured at baseline, and in the morning subsequent to the treatments (with AS and TT) and wash out.

\section{Patient's preference}

At the endo of the trial the patients were asked which treatment was preferred between AS and TT.

\section{Blood glucose control}

Blood glucose was controlled at home by the patients himself as normal routine monitoring. During the treatment, data out of the usual levels of glucose had to be communicated to the investigator.

\section{Products under testing}

Two products were tested; aldiamed ${ }^{\circledR}$ spray (AS) and Tonimer lab throat spray (TT) consisting of ingredients of sea origin. The products were given three times/day 3 days as follows: at $8 \mathrm{am}, 2 \mathrm{pm}$, and $10 \mathrm{pm}$. 
Both AS and TT were administered each time using 3 puffs (consisting of $0.15 \mathrm{~mL}$ each; total $0.45 \mathrm{~mL} /$ administration). Each subject was given 1 box aldiamed ${ }^{\circ}$ spray and 1 box of Tonimer throat spray. Bottles were blank with no identification a part of the labelling A or B (respectively for AS and TT).

\section{Compliance}

The compliance was measured calculating the difference of the weight of the spray for AS and TT.

\section{Statistical analysis}

\section{Sample size}

The sample size was calculated on the base of the xerostomia measure taken on the second phase of the study. The mean value was $4.2 \pm 0.75$ (SD) and a clinically valid reduction was expected to reach at least the mean value of $3 \pm 0.74$ (consistent with about $30 \%$ reduction). Considering $\alpha 0.05$ and $1-\beta 0.90$ the number of subject needed was 13 . The presence of possible drop in the reason of $20 \%$ was suggesting enlisting at least 16 cases. The allocation to the arms was determined by computer randomization.

\section{Data analysis}

For each variable was calculated the mean value $(M)$ and the standard deviation (SD); the differences among treatment was determined using a variance analysis for crossover studies. Since xerostomia was measured using scores, the crossover analysis was confirmed with the Schuirmann's two one-side test procedure. In case of absence of carryover effects between the two arms of the crossover, the data of the two products will be pooled and compared with the respective baseline values using the Wilcoxon signed rank test. Correlation will be calculated among all the variables using Density Ellipses analysis. All the statistical procedure was calculated with MacBook Pro using JMP13 programs of SaS institute Inc. and NCSS 13.

\section{Blindness}

The measures (xerostomia, salivary flow, and SAT were taken by investigators that were not aware about the type of treatment. The statistician received the data in an excel file where the treatment was reported as A or B.

\section{Results}

Twenty-one subjects were interviewed and 16 only participated the crossover study as reported in (Figure 1). The reason of exclusion was xerostomia $<2$ (four cases) and BMI was $>30 \mathrm{Kg} / \mathrm{m}^{2}$ ( 1 case).

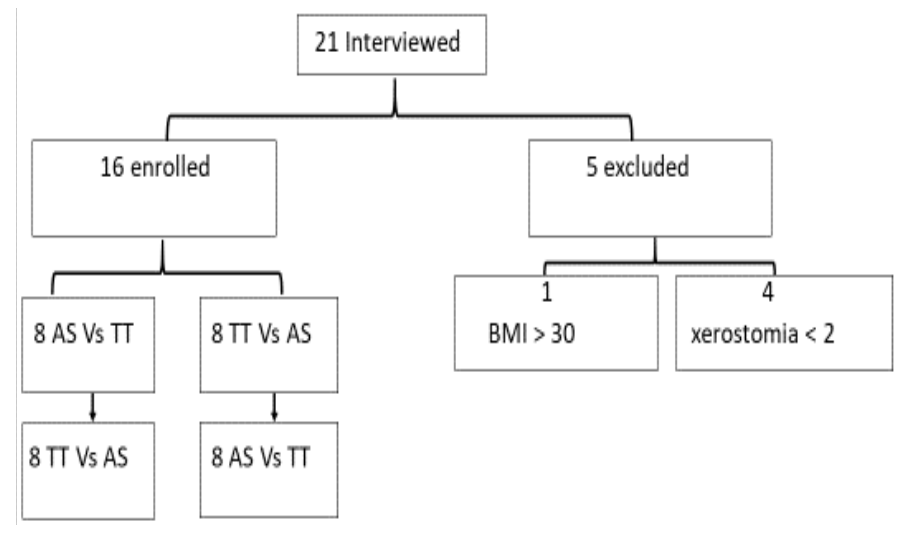

Figure 1. Registry crossover flow chart
Table 1. General characteristics of the patients: Mean \pm SD or frequency

\begin{tabular}{|l|l|l|}
\hline Variables & Measure & Value \\
\hline Age & years & $63.6 \pm 7.19$ \\
\hline Gender & & $9 \mathrm{M} \quad 7 \mathrm{~F}$ \\
\hline Weight & $\mathrm{Kg}$ & $73.8 \pm 8.63$ \\
\hline Height & $\mathrm{m}$ & $1.71 \pm 0.069$ \\
\hline BMI & $\mathrm{Kg} / \mathrm{m}^{2}$ & $25.1 \pm 1.92$ \\
\hline Years of Diabetes Type II & Years & $3.1 \pm 1.75$ \\
\hline Concomitant diseases & & $\mathrm{N}$ \\
\hline Hypertension & & 2 \\
\hline Dyslipidemia & & 1 \\
\hline Gastrointestinal diseases & & 2 \\
\hline Chronic venous insufficiency & & 1 \\
\hline Central nervous system & & 1 \\
\hline
\end{tabular}

Table 2. Mean values $\pm \mathrm{SD}$ at baseline, after AS and TT and after washing out

\begin{tabular}{|c|c|c|c|c|c|c|}
\hline \multirow{2}{*}{$\begin{array}{c}\text { Variable/ } \\
\text { symptom }\end{array}$} & \multirow{2}{*}{ Measure } & \multirow{2}{*}{ Baseline } & \multirow{2}{*}{ Wash out } & \multicolumn{2}{|c|}{ Treatments } & Pa $^{\mathbf{a}}$ \\
\cline { 5 - 7 } & & & AS & TT & AS Vs TT \\
\hline $\begin{array}{c}\text { Xerostomia } \\
\text { Salivary } \\
\text { Flow }\end{array}$ & $\mathrm{mL} / \mathrm{min}$ & $0.37 \pm 0.154$ & $0.41 \pm 0.150$ & $0.39 \pm 0.154$ & $0.41 \pm 0.136$ & $>0.05$ \\
\hline SAT & $\mu \mathrm{mol} / \mathrm{L}$ & $3290 \pm 505.3$ & $3387 \pm 480.9$ & $2496 \pm 285.2$ & $3377 \pm 461.9$ & $<0.001$ \\
\hline
\end{tabular}

${ }^{a}$ Crossover analysis

All the subject completed the study and no complaint of side effect was given for any of the products.

The general characteristics of the patient are reported in Table 1.

The results of the symptoms modification are reported in Table 2.

No modification of the usual blood glucose levels was reported by the patient in any phase of the research.

There was not a significant carryover effect between the Baseline and Wash out period for any of the variables/symptoms considered. The saliva flow was not modified by any of the treatment, whereas for xerostomia and SAT a clear statistically significant difference was detected. The difference in the xerostomia modification was confirmed by the Schuirmann's test. Any correlation was found among the variables.

Due to the absence of carryover effect the observations of the two groups could be pooled and compared to the relative baseline values (Table 3). The activity of AS was shown to be significantly superior to TT

Table 3. Analysis of the pooled data after AS and TT: percentage reduction and Wilcoxon Signed Rank test

\begin{tabular}{|c|c|c|c|}
\hline Variable & $\begin{array}{c}\text { AS \% } \\
\text { Vs Baseline }\end{array}$ & $\begin{array}{c}\text { TT \% } \\
\text { Vs Baseline }\end{array}$ & Wilcoxon test \\
\hline Xerostomia & -59.6 & -2.9 & $\mathrm{p}<0.001$ \\
\hline Salivary flow & +5.1 & +11.1 & $\mathrm{p}>0.05$ \\
\hline SAT & -24.2 & +2.7 & $\mathrm{p}<0.001$ \\
\hline
\end{tabular}

\section{Discussion}

The present research has some limitation because the products were used for a very short period of time.

Despite the treatment schedule was identical for the two products, a longer treatment should be needed for a better definition of AS activity. However, some indication can be drawn from this clinical 
pharmacology study, such as the lack of any correlation among the variables that have been studied. These aspects indicate that each symptom is independent and may express a particular aspect of the oral discomfort.

Xerostomia is frequently present in patients suffering from diabetes Type II and although it has to be considered a subjective symptom, in these patients it may objectively be associated with impaired functioning and by the polyuria determined by the poor control of blood glucose.

Frequently, these patients are under polytherapy for concomitant diseases taking drugs that are known to cause xerostomia, which is determining a continuous discomfort such that its relief is an important achievement to improve the daily living.

The results of the present study indicate that in these patient's xerostomia can be counteracted without changing the salivary flow and without the burden of a further pill intake.

The activity of the AS on this symptom was very evident and attributed to its capability to increase the wettability of the oral mucosa due to the presence of humectants such as the propylene glycol.

The reduction of SAT mirrors the improvement of the oral inflammatory condition and has a more complex explanation. In the mouth some pathogenic bacteria cause the production of reactive species, mainly hydrogen peroxide $\left(\mathrm{H}_{2} \mathrm{O}_{2}\right)$ that is generated to prevail over the other species. In part $\mathrm{H}_{2} \mathrm{O}_{2}$ is also normally produced by mucosal cells to control the bacterial growth.

The consequence is that antioxidants ( $\mathrm{AOx}$ ) are produced by the epithelial/mucosal cells to protect themselves from the oxidation. However, in case of $\mathrm{H}_{2} \mathrm{O}_{2}$ overproduction (by bacteria and oral cavity cells) the physiological AOx availability is not sufficient to protect from the oxidative stress, and the reactivity of the damaged tissue end up with the production of inflammatory cytokins, that induce vasodilation and consequent extravasation of AOx from vessels of the oral cavity. This phenomenon is consistent with the increase of SAT over 2.500 $\mu \mathrm{mol} / \mathrm{L}$.

The activity of some artificial saliva on oral inflammation have been already described [13] and for AS may depend on the presence two antimicrobial agents, lactoferrin and lysozyme that are acting on those bacteria producing $\mathrm{H}_{2} \mathrm{O}_{2}$. The mucoadesive polymers present in the product allow to maintain the residence time of these natural antimicrobial agents. Furthermore, the same polymers cover the oral surface, and allow the restoring of the damaged epithelium.

One peculiar aspect of this formulation is the presence of Aloe Vera which is known for the antioxidant, anti-inflammatory [14], and also antibacterial activity, the latter particularly as a gel [15]. These activities are consistent with the content of the acemannan in Aloe vera. This polysaccharide has many properties that can be useful for the oral cavity protection [16], since it was documented its activity in the acceleration of the healing and also for the osteogenetic capacity. In experimental studies was shown that acemannan reduces the pulp inflammation [17] and stimulates the dentin formation [18].

\section{Conclusions}

The treatment with aldiamed ${ }^{\oplus}$ spray was reducing xerostomia and oral inflammation in patient suffering from diabetes Type II.

\section{Acknowledgements}

The authors are gratefull to prof Martino Recchia (University of Lugano) who was making all the statistical evaluation and determined the sample size.

\section{Competing interest}

None

\section{Funding}

No funding was requested or given for this registry study. Certmedica International $\mathrm{GmbH}$, Magnolienweg 17, 63741 Aschaffenburg, Germany was providing the artificail saliva spray (aldiamed ${ }^{\circ}$ ) free of charge, whereas the Tonimer throat spray was provided by the investigators. The patients were not paid for participating in the trial, but received free treatments.

\section{Ethical Committee}

Despite this Registry study was not needing any official ethical approval since no invasive tests were applied, it was submitted to IAPSS (Internation Agency for Pharma-Standard Supplement- PE Italy) that gave its approval.

\section{Authors Contribution}

UC, GB, were responsible for designing the research, GB was conducting the trial; the statistical analysys was contucted by an external office (prof Martino Recchia); UC and GB wrote the paper.

\section{References}

1. López-Pintor RM, Casaňas E, Gonzáles-Serrano J (2016) Xerostomia, hyposalivation, and salivary flow in diabetes patients. J Diab Res 2016: 4372852 [Crossref]

2. Bajai S, Prasd S, Gupta A, Singh VB (2012) Oral manifestation in type II diabetes and related complications. Indian J Endocrinol Metabol 16: 777-779 [Crossref]

3. Hoseini A, Mirzapour A, Bijani A, Shirzad A (2017) Salivary flow rate and xerostomia in patients with type I and type II diabetes mellitus. Electr Phys 9: 5244-5249 [Crossref]

4. Malicka B, Kaczmarek U, Skośkiewicz-Malinowska K (2014) Prevalence of xerostomia and the salivary flow rate in diabetic patients. Adv Clin Exp Med 23: 225-233 [Crossref]

5. Ittichaicharoen J, Chattipakorn N, Chattipacorn SC (2016) Is salivary gland function altered in noninsulin-dependent diabetes mellitus and obesity-insulin resistance? Arch Oral Biol 64: 61-71 [Crossref]

6. Lin CC, Sun SS, Kao A, Lee CC (2002) Impaired salivary function in patients with noninsulin-dependent diabetes mellitus with xerostomia. J Diabetes Complications 16 : 176-179 [Crossref]

7. Chávez EM, Borrel LN, Taylor GW, Ship JA (2001) A longitudinal analysis of salivary flow in control subjects and older adults with type 2 diabetes. Oral Surg Oral Med Oral Pathol Oral Radiol Endod 91: 166-173 [Crossref]

8. Dalodom S, Lam-ubol A, Jeanmaneechotechai S (2016) Influence of oral moisturizing Jelly as a saliva substitute for the relief of xerostomia in elderly patients with hypertension and diabetes mellitus. Ger Nurs 37: 101-109 [Crossref]

9. Engelhart K, Popescu A, Bemhardt J (2016) Using mid infrared technology as new method for the determination of the dwell time of salivary substitutes on three dimensional gingiva models. BMC Ear, Nose, Throat Disorders 16: 6 [Crossref]

10. Hahnel S, Etti T, Gosau M (2010) Arch Oral Biol 55: 391-396.

11. Cornelli U, Belcaro G, Nardi GM (2010) Action of an antioxidant complex on the antioxidant power of saliva. Pan Med 2: 69-73 [Crossref]

12. Benedetti S, Primiterra M, Finco A (2014) Validation of a patented method to determine the antioxidant capacity of human saliva based on the reduction of iron: the SAT test. Clin Lab 60: 475-482 [Crossref]

13. Kang M, Park H, Jun J (2017) Facilitated saliva secretion and reduced oral inflammation by a novel artificial saliva system in the treatment of salivary hypofunction. Drug Des Dev Ther 11: 185-191[Crossref] 
14. Nair GR, Naidu GS, Jain S (2016) Clinical effectiveness of Aloe Vera in the management of oral mucosal diseases: a systemetic review. J Clin Diag Res 10: ZE01ZE07 [Crossref]

15. Ehsani M, Amin Marashi M, Zabini E (2013) A comparison between antibacterial activity of propolis and Aloe vera on Enterococcus faecalis (an In vitro study) Int J Mo Cell Med 2: 110-116 [Crossref]

16. Sierra-Garcia GD, Castro-Rios R, Gonzáles- Horta A (2014) Acemannan, an extracted polysaccharide from Aloe vera: a literature review. Nat Prod Commun 9: 1217-1221 [Crossref]
17. Songsiripradubboon S, Kladkaew S, Trairatvorakul C (2017) Stimulation od dentin regeneration by using acemannan in teeth with lipopolysaccharide-induced pulp inflammation. J Endod 43: 1097-1103 [Crossref]

18. Jittapiromsak N, Sahawat D, Banlunara W (2010) Acemannan, an extracted product from Aloe vera, stimulates dental pulp cell proliferation, differentiation, mineralization and dentin formation. Tissue Eng Part A 16: 1997-2006 [Crossref]

Copyright: $@$ 92018 Cornelli U. This is an open-access article distributed under the terms of the Creative Commons Attribution License, which permits unrestricted use, distribution, and reproduction in any medium, provided the original author and source are credited. 